\title{
A novel single-use SU-8 microvalve for pressure-driven microfluidic applications
}

\author{
J. Miguel Moreno, José M. Quero \\ Escuela Superior de Ingenieros, University of Seville, Seville, Spain \\ E-mail: jmmoreno@gte.esi.us.es, quero@us.es
}

\begin{abstract}
A novel microfluidic single-use valve for fluid injection and extraction in pressuredriven applications is presented in this paper. The device consists in a thin SU-8 membrane crossed by a resistor that withstands a mechanical stress induced by a pressure difference. When the resistor heats up the membrane, the SU-8 fracture strength drastically decreases causing the valve activation. This device has been designed, fabricated using inexpensive SU-8 and Printed Circuit Board technologies, and finally characterized. The hybrid thermalmechanical microvalve operation principle has been demonstrated and experimental results have shown the device characteristics and performance. Specifically, this design was functional at pressures of $0.8 \mathrm{MPa}$ and opened in less than 3.2 seconds with an applied power of $280 \mathrm{~mW}$. The simple fabrication process and the absence of moving mechanical parts have made the valve suitable for large-scale integration in Lab-on-Chip microfluidic platforms.
\end{abstract}

\section{Introduction}

Since the rapid expansion and development of microfluidics during the last years, microvalves and micropumps have become essential elements in microscale fluid handling. The requirements for precise control of fluid flow in a widely range of areas from biomedical and drug delivery to automotive, aeronautics and fuel cell systems, has motivated the development of this microfluidic devices using microelectromechanical systems (MEMS) technology [1]. During the last two decades, MEMS and microfluidics research have been largely encouraged by the realization of a fully integrated microfluidic platform, such as micro total analysis systems ( $\mu$ TAS) [2] or Lab-on-chip (LOC) [3].

These devices are potentially important in biotechnology, clinical diagnostics and medical applications, performing the functions of a bio-laboratory in a single chip. Several Lab-on-chip devices have been reported in the literature by different research groups [4-6]. The microscale platform not only makes possible to work with small volume of fluids but also has many distinct advantages such as reduced consumption of expensive chemical reagents, faster analysis times as well as increased sensitivity and reliability [7]. Microvalves are essential components in such devices for portable chemical analysis, sample transport, drug delivery or mixing, but their actual lack of reliability have become one of the main obstacles that have delayed the successful miniaturization and commercialization of fully integrated microfluidic systems.

Microvalves are used not only to provide fluid flow regulation in microchannels but also to seal liquids, gases or vacuums and to switch between open and closed flow. One way of classifying microvalves is through the actuation mode, where two main types can be found: passive and active valves. Passive valves have no actuation due to the open/closed states come from the internal energy 
of the fluid. On the other hand, active microvalves require external energy to operate, presenting a structure formed by a closing element and an actuator. Active valves can either be categorized by their initial working state or by their actuation principle. The three initial working states are normally closed, open or bistable, and the main actuation methods are pneumatic, thermopneumatic, thermomechanical, electrostatic, piezoelectric, electromagnetic, electrochemical and phase change. A detailed classification of microvalves has been reported in the literature by several authors $[8,9]$.

However, high manufacturing costs, fragility and lack of reliability of these devices still present a serious obstacle to integration. Specifically, traditional microvalves are fabricated in silicon, but high costs and limited accessibility of silicon processing has driven interest in exploring other materials and alternative technologies. During the past decade, polymers such as SU-8 and PDMS have emerged as materials of choice for many microfluidic devices and LOC platforms, offering the advantages of inexpensive and simple fabrication processes using rapid prototyping $[10,11]$.

In this respect, SU-8 technology has been a widely researched and developed area. SU-8 is an epoxy-based negative photoresist, whose properties make it a useful and cheap alternative for many microfluidic applications [12]. It can be patterned using standard mask processes with very thin films and nearly vertical sidewalls profiles [13]. Furthermore, the resist is transparent so the fluids can be sensed using an optical approach and inspected during experiments. Biocompatibility and biofouling of SU-8 have been previously evaluated and reported, presenting suitable results for biomedical applications [14].

Another technology that is becoming important in miniaturized fluid components is Printed Circuit Board Microsystems (PCBMEMS), which combines mechanical, electronic and fluidic devices by adding some new steps on common PCB fabrication process [15]. The main advantage of these devices lies in high integration with inexpensive materials, making possible to place all the components necessary for sensing, analyzing and controlling the fluids onto the same PCB.

In some microfluidic applications it is sufficient to open a flow channel only once in order, for example, to trigger a mixing reaction in a microchamber or to deliver some fluid sample at a certain time. For such one-shot actuations, a disposable microvalve design can minimize reliability problems and manufacturing cost. Single-use microvalves contain a destructible element that is commonly activated by thermal or chemical actuation. The benefits of thermal techniques over chemical ones are simplicity and safety, due to the absence of risky or toxic reagents.

Several authors have reported different contributions to this area, developing single-use microvalves with diverse operation principles and materials. Thermally actuated paraffin microvalves [16] and destructible membranes coated with low melting point alloys [17] can be found in the literature. Similar studies oriented to high gas pressure applications or about integration of air-bursting detonators are reported in $[18,19]$ respectively. However, the main disadvantages of thermal actuation are principally related to high energies required for valve activation and slow time responses when compared to many active microvalves.

The purpose of this work is to develop a novel single-use microvalve designed to overcome the common limitations presented in traditional microfluidic devices. The presented device is activated combining two simultaneous and different phenomena, thermal and mechanical, minimizing the energy consumption required to activate the device. The valve design has been conceived to control the fluid flow in pressure-driven microfluidic applications, where the fluid motion energy is contained in a pressurized tank. Considerable effort has been directed towards minimizing the device cost, improving its integration and simplifying the fabrication process. Consequently, inexpensive materials like SU-8 and PCB have been selected.

This paper is organized as follows. In section 2, device hybrid operation principle is presented and theoretical pressure and temperature effects over the membrane are studied separately. The following section shows the fabrication process and the device finally implemented. Experiments and results are discussed in section 4. Finally, conclusions are presented in section 5. 


\section{Operation principle}

A schematic of the microvalve concept is shown in figure 1. It consists of a thin SU-8 membrane crossed by a resistor element. A pressure difference is fixed between both sides of the membrane in order to cause a mechanical stress over the structure. This stress will produce a breakage on the membrane when an electric current is supplied through the resistor element. The purpose of this resistor is to weaken the membrane by thermal power, acting as a trigger for the activation. In absence of electric current the membrane will remain as a solid barrier, withstanding the pressure difference between both sides. Therefore, the microvalve actuation is achieved by a combination of pressure and temperature actions.

When the membrane is broken, a fluid contained in the higher pressure side will flow to the other side of the membrane in order to reach the equilibrium of pressures. Membrane dimensions are studied and discussed in detail in the following subsection to achieve a correct operation of the device.

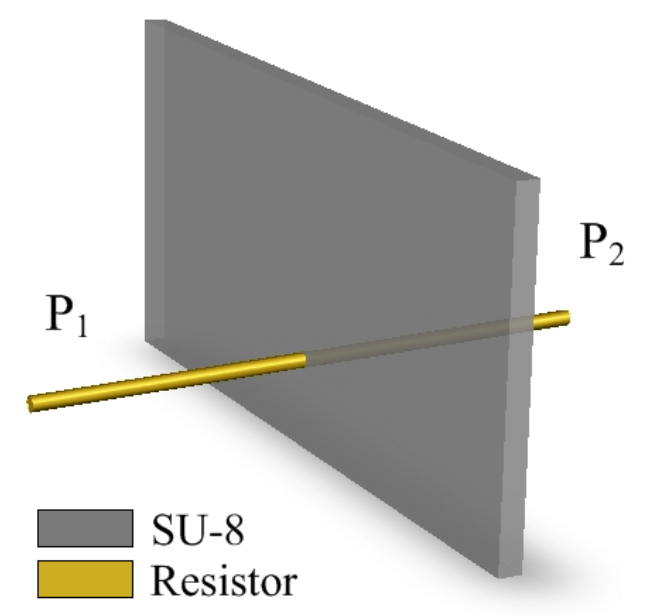

Figure 1. Schematic of the microvalve concept.

\subsection{Mechanical actuation}

An essential component in microvalve design is the SU-8 membrane, in charge of withstanding the mechanical stress produced by the pressure and sealing completely the fluid path. Dimensions of this membrane will play a fundamental role in the thermal power required as well as the time taken for the valve to operate. Due to fabrication process the membrane geometry will be restricted to square or rectangular section. According to the mathematical approximation reported by [20], the maximum bending stress, $\sigma_{\max }$, generated by a uniform pressure $P$ on a clamped rectangular plate with a width $L_{y}$, a length $L_{x}$ and a thickness $h$ can be expressed as:

$$
\sigma_{\max }=c \frac{P}{\left(h / \min \left(L_{y}, L_{x}\right)\right)^{2}}
$$

where the coefficient $c$ is calculated by the polynomial least-square curve-fitting as a function of $L_{y}$ and $L_{x}$.

This formula is valid for most materials that have Poisson's ratios around 0.3. Previous works have reported a 0.22 Poisson ratio for the SU-8 polymer [12], assuming then a similar membrane behavior to expression (1) when a real pressure is applied. In order to verify the theoretical analysis before determining the appropriate dimensions for the membrane, software tool Coventorware was used to simulate the phenomenon involved in valve operation. A rectangular membrane with a width of 500 
$\mu \mathrm{m}$, a length of $1000 \mu \mathrm{m}$ and a thickness of $40 \mu \mathrm{m}$ was selected to study the maximum bending stress applied by a pressure of $0.7 \mathrm{MPa}$, in order to localize possible breakage points. Simulation results are shown in figure 2.

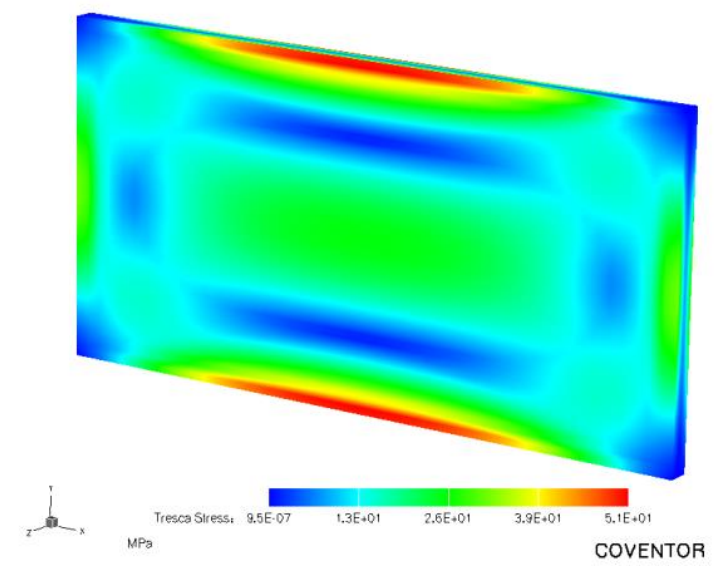

Figure 2. Bending stress produced by a pressure of $0.7 \mathrm{MPa}$ applied over a $500 \times 1000 \times 40 \mu \mathrm{m}^{3} \mathrm{SU}-8$ membrane.

The simulation illustrates the zones where the bending stress is maximum, located in the center point of both long edges near bottom and top surfaces of the membrane. Thereby, the optimum place to cross the membrane with the resistor will be on its low-center zone near the substrate, in order to heat the weakest area. The simulation exhibited a maximum bending stress of $51 \mathrm{MPa}$, while the calculated value in (1) was approximately $55 \mathrm{MPa}$, validating the theoretical expression.

According to (1) $\sigma_{\max }$ will be thickness-to-width aspect ratio quadratic dependent. This fact establishes the membrane aspect ratio and the pressure applied as main parameters involved in mechanical actuation. According to previous authors, the SU-8 fracture strength $\sigma_{f}$ has been reported as $62.9 \mathrm{MPa}$ at room temperature, assuming optimum processing conditions for the resist [21]. Thus, high membrane aspect ratios or very high pressures will be required to perform a mechanical breakage without any thermal contribution. The maximum theoretical bending stress in the membrane as a function of aspect ratio and pressure is reported in figure 3 .

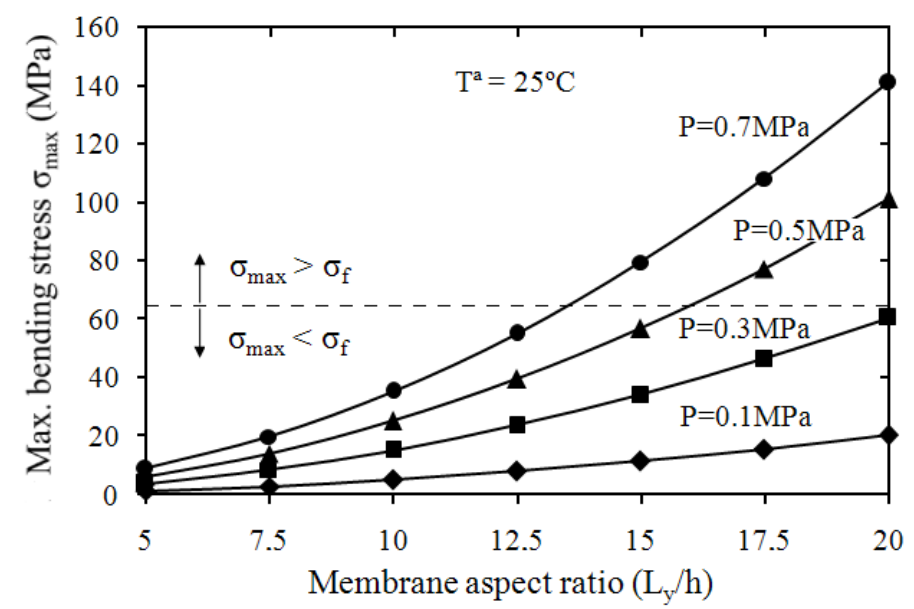

Figure 3. Theoretical bending stress as a function of membrane aspect ratio and pressure applied. Dashed line shows the SU-8 fracture strength under optimal conditions. 
Experimental assays with different membrane dimensions were carried out in section 4 to confirm these limitations, where an increment of resistor temperature was necessary to achieve such strict breakage conditions.

\subsection{Thermal actuation}

In this section, the heating of the SU-8 membrane due to the temperature transfer of the resistor element is discussed, when the pressure applied to the membrane is assumed to be negligible. A gold microwire commonly used in wire bonding technology for connecting microchips to external pads in semiconductor device fabrication was selected as a resistor. This proposal maximizes the thermal power generated-to-electric current ratio due to the small cross-sectional area of the wire. In our study the wire presented a $25 \mu \mathrm{m}$ diameter section.

Different designs actuated by thermal phenomenon have been considered to activate the valve, comparing copper PCB microlines to gold wires. When metal is heated up, conduction phenomenon to air and also to PCB substrate plays an important role due to thermal power losses. Simulation and experimental results determined that isolation between metal and substrate reduces power losses and concentrates the heat transferred to the membrane. For this reason, a gold wire was bonded between copper lines with a certain curvature, in order not to be in contact with PCB substrate.

When the wire temperature increases by current effect, a radial heat distribution will propagate from the wire surrounding area with higher temperature to the membrane edges that initially are at ambient temperature. This effect was simulated with Coventorware as is shown in figure 4.



(a)

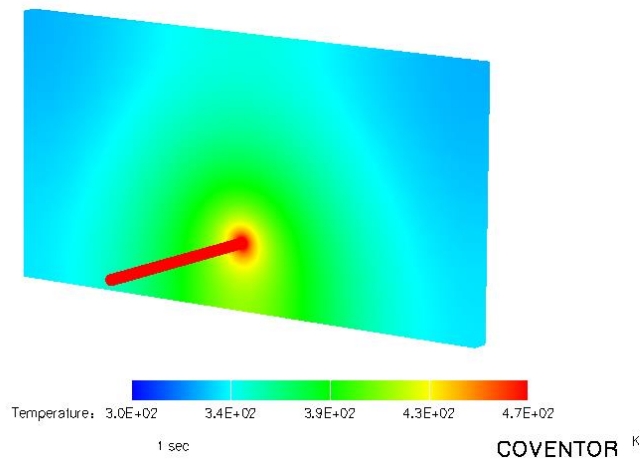

(c)

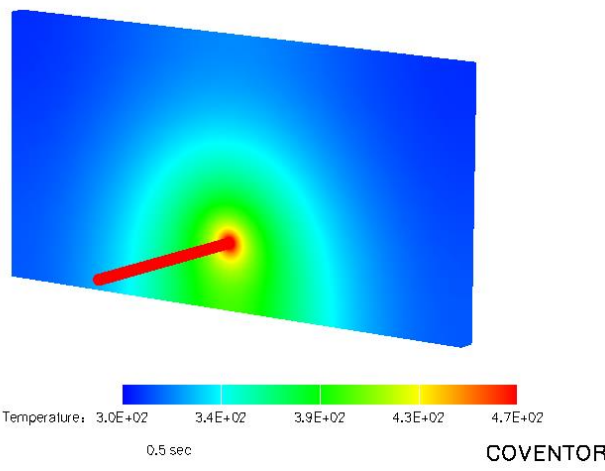

(b)

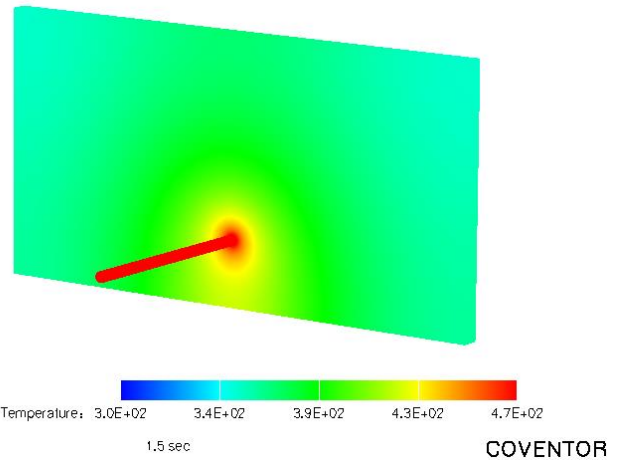

(d)

Figure 4. Thermal conduction through a $25 \mu \mathrm{m}$ section diameter gold wire which crosses a $500 \times$ $1000 \times 40 \mu \mathrm{m}^{3} \mathrm{SU}-8$ membrane when (a) $\mathrm{t}=0.1 \mathrm{~s}$, (b) $\mathrm{t}=0.5 \mathrm{~s}$, (c) $\mathrm{t}=1 \mathrm{~s}$ and (d) $\mathrm{t}=1.5 \mathrm{~s}$. Wire temperature selected for simulations was $200{ }^{\circ} \mathrm{C}$. 
With this in mind, the theoretical temperature reached in the wire $T_{\text {int }}$ can be obtained as a function of the heat generated $Q$ by Joule effect, which depends on the external current supplied $I$ flowing through a conductor of electrical resistance $R$ for a time $t$. The thermal conduction through the wire, assumed as a cylinder, can be mathematically expressed as follows:

$$
\frac{Q}{H}=\frac{I^{2} \cdot R \cdot t}{H}=\frac{T_{\mathrm{int}}-T_{e x t}}{\ln \left(r_{\text {ext }} / r_{\mathrm{int}}\right) / 2 \pi k}=\frac{\Delta T}{R_{c}}
$$

where $H$ is the wire length that crosses the membrane, $\Delta T$ is the temperature gradient between the wire perimeter $T_{i n t}$ and the membrane edges $T_{\text {ext }}$ (initially assumed as ambient temperature), $r_{\text {ext }}$ and $r_{\text {int }}$ are the external radius from the heat source to the membrane edge and the internal radius to the wire border, respectively, $k$ is the SU-8 conductivity and $R_{c}$ is the thermal resistance. According to equation (2), the wire temperature can be solved as follows.

$$
T_{\mathrm{int}}=T_{e x t}+R_{c} \cdot \frac{Q}{H}
$$

With this equation the theoretical wire temperature as a function of the current supplied is illustrated in figure 5 .

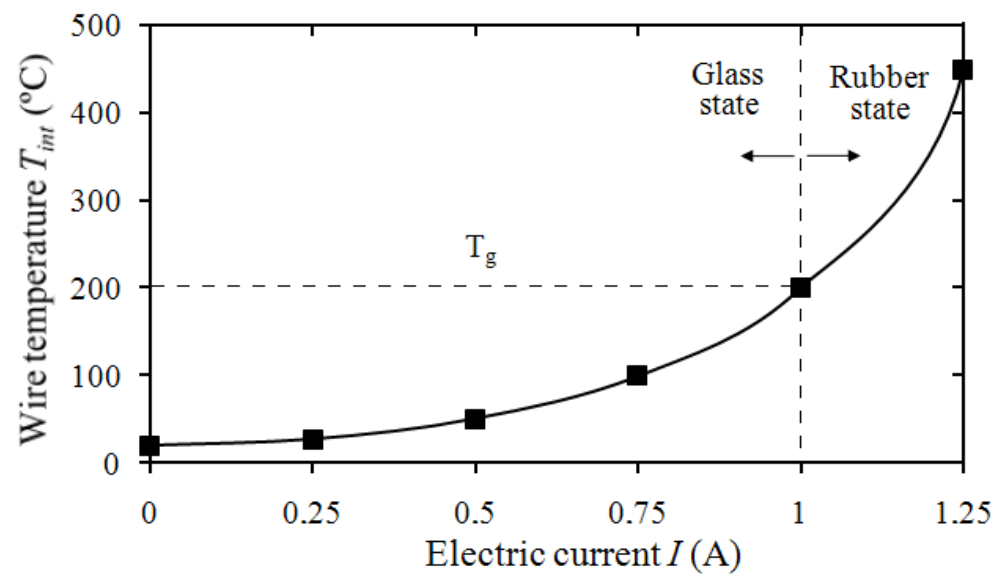

Figure 5. Theoretical temperature as a function of the current supplied to the wire at steady state. Dashed vertical line shows the current necessary to reach the SU-8 glass transition temperature, $T_{g}$.

Previous studies reported that the SU-8 glass transition temperature $T_{g}$ is approximately $200{ }^{\circ} \mathrm{C}$ [22]. From this point the polymer is in its rubbery state, lending softness and flexibility to the membrane. At higher temperatures the SU-8 starts melting down until degradation temperature is reached, located near $380^{\circ} \mathrm{C}$. It is important to underline that the present study has been carried out without taking into account another heat conduction effects in the membrane like convection or radiation to air, resulting in an effective reduction of the temperature showed in figure 5 during real assays. In addition to this, SU-8 Young's modulus linearly decreases approximately from $50{ }^{\circ} \mathrm{C}$ to 150 ${ }^{\circ} \mathrm{C}$, as is reported in [23]. In this temperature range, SU-8 Young's modulus will be nearly one order of magnitude smaller, affecting the membrane visco-elastic properties. However, the numerical results and conclusions resulting from this theoretical approximation will help us in the discussion of the experiments carried out in section 4 . 


\subsection{Hybrid actuation}

In the device presented, high power consumptions commonly associated to thermally actuated microvalves has been overcome by setting a differential pressure between the chamber and the microchannel in order to help the membrane destruction by mechanical stress. The combined actuation of thermal and mechanical principles cause a synergistic effect, drastically reducing the energy needed for activation and decreasing the SU-8 fracture strength $\sigma_{f}$ due to temperature effect. Besides this, SU8 Young's modulus reduction results in an increment of material elasticity and consequently in a raise of membrane displacement when pressure is applied.

According to the physic mechanisms involved in the device operation, two different mathematical models can be assumed as a function of the heat reached in the membrane. On one hand, when this temperature is lower than $T_{g}$ the membrane behaviour will be similar to a SU-8 rectangular plate clamped on all edges under a uniform pressure, with a fracture strength also thermally dependent. On the other hand, when membrane temperature exceeds SU-8 glass transition the model will be assumed as a viscous cylinder around the gold wire subjected to a uniform pressure.

The phenomena concerned in this mixed actuation principle leads to a complex mathematical model with very hard assumption. For this reason, an experimental study has been carried out to determine the behaviour of the operation process.

\section{Fabrication}

The implementation of the microvalve is simple to be carried out and employs inexpensive materials due to the use of SU-8 and PCBMEMS technologies. Integration of microfluidics and classical PCB electronic connections in a common substrate is achieved by adding some new steps in the fabrication process. Membrane fabrication, gold wire alignment accuracy and final bonding and enclosure with the cover board are critical steps in the complete fabrication process flow. The device design, materials involved in fabrication and process flow are detailed as follows.

\subsection{Design}

An overhead and cross-section view of the complete device is shown in figure 6. It is formed by a SU8 structure composed of a circular chamber and a microchannel separated by the membrane. The gold wire that crosses the membrane is connected to two independent copper lines, patterned on a common printed circuit board substrate. Two small orifices are performed in the chamber and the microchannel, acting as a connector to an external pump to set up the operation pressure, and to allow the fluid flow by means of an input/output fluid port, respectively. A transparent board is bonded to the structure as a cover, allowing the valve to be inspected during pressure and breakage experiments.

Pressure on chamber and microchannel will determine the fluid flow direction, establishing a reversible valve operation mode. If the microchannel is set at atmospheric pressure and chamber pressure is higher the device will operate as a microinjector, whereas with lower pressure than atmospheric, the valve will work in microextractor mode, forcing the fluid contained in the inlet/outlet port through the channel towards the chamber.

Dimensions of the chamber and the microchannel have been calculated to ensure the microchannel filling with fluid, which flows through the input/output port after activation in microextractor operation mode with the chamber pressurized at $10 \mathrm{kPa}$. The energy stored in the chamber will drive the fluid through the channel. The microchannel length was $8 \mathrm{~mm}$ with a square section of $500 \times 500$ $\mu \mathrm{m}^{2}$, and the circular pressure chamber was designed with a radius of $1.8 \mathrm{~mm}$ and a total internal volume of $5 \mu \mathrm{L}$. Chamber and microchannel SU-8 walls as well as copper lines presented a width of $500 \mu \mathrm{m}$. The total dimensions of the device were $4 \times 12 \times 5 \mathrm{~mm}^{3}$. The purpose of the structure designed is to validate the membrane hybrid operation principle, visualizing the fluid flow when the microvalve is activated during experiments. 
Fluid

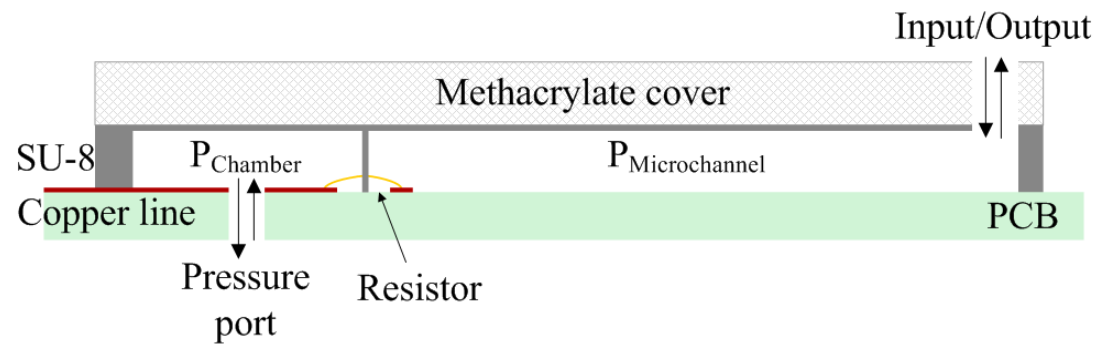

(a)

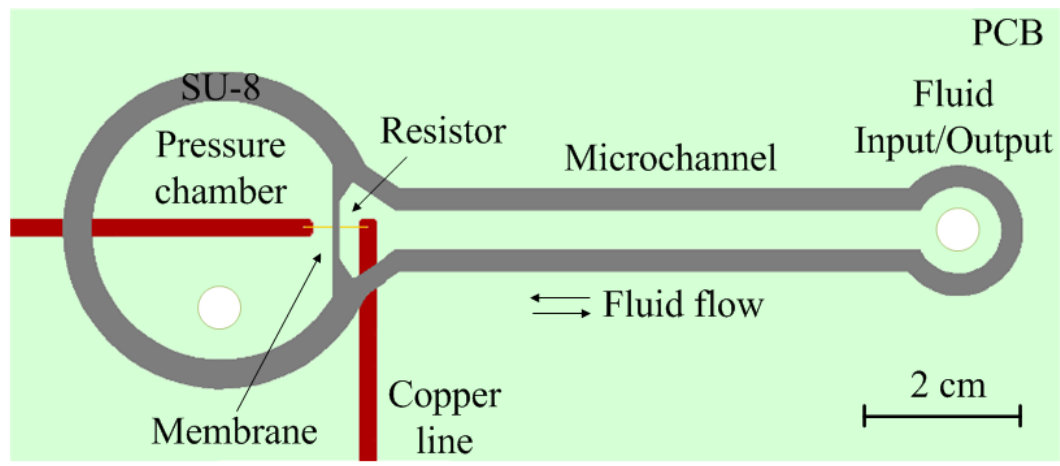

(b)

Figure 6. Cross-section view (a) and overhead view (b) of the microvalve design. Pressure in the chamber is regulated through a PCB orifice.

\subsection{Materials}

Following the basic idea of PCB-based technology, a conventional single-sided copper-plated rigid material (FR4) was used as a substrate. The thickness of the copper layer and the total thickness of the PCB are selectable, however commercially standard $35 \mu \mathrm{m}$ and $1500 \mu \mathrm{m}$ copper and FR4 respectively were chosen.

SU-8 2150 photoresist (supplied from Microchem Corporation) was applied to form the basic device structure: pressure chamber, membrane and microchannel. Other materials were previously suggested to compose the membrane, like PDMS elastomer, Paraffin or epoxy resists. Their mechanical and thermal properties are critical for the microvalve operation, due to the required capabilities of supporting high pressures and reaching its glass transition point at relatively low temperatures, respectively. In experimental tests, these materials showed low mechanical endurance or excessive low glass transition temperatures, resulting unsuitable for the proposed device. In addition to this, the width control of the membrane during fabrication process was better achieved by means of SU-8 technology. Therefore, SU-8 2150 was finally selected in order to build high sidewall profiles for the membrane, accomplishing a monolithic structure without additional fabrication steps and exhibiting suitable mechanical and thermal properties.

Epo-Tek 302-3M resist (Epoxy technology Inc.) reported in previous investigations [24] was replaced with SU-8 2025 photoresist as adhesion layer between the structure and the transparent cover, resulting into a stronger linking. A $3 \mathrm{~mm}$ thickness methacrylate cover was selected to completely seal the device ensuring pressure chamber enclosure and avoiding possible leakages. This transparent cover allowed the valve to be inspected during pressure and breakage experiments. 


\subsection{Process flow}

The complete microvalve fabrication procedure is illustrated in figure 7 and is described as follows.

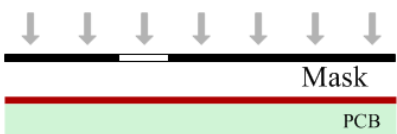

a) UV exposure

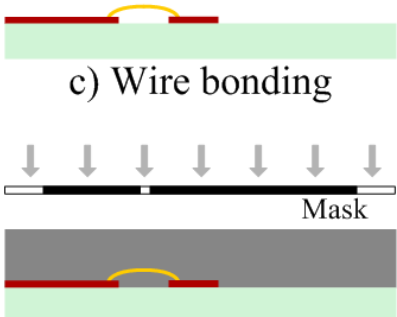

e) UV exposure

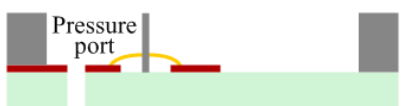

g) Drilling

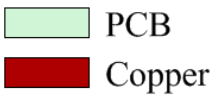

Copper lines

b) Wet etching

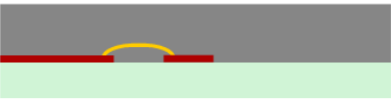

d) SU-8 deposition

Pressure
chamber Microchannel

f) SU-8 Patterning

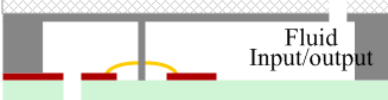

h) Cover bonding

Figure 7. Microvalve fabrication process flow.

The fabrication process started with an UV light exposure over a $5.5 \mathrm{~cm}^{2}$ square PCB substrate during 2.5 minutes. After UV lithography with the corresponding mask, the exposed areas were developed by wet etching obtaining the connections where the gold wire is subsequently bonded. Several adhesion tests involving SU-8-copper and SU-8-FR4 determined that the stronger cohesion was achieved depositing SU-8 over a copper layer. For this reason, the mask was designed with copper areas under the complete valve structure, avoiding short circuits with the copper lines in charge of supplying the electric current to the wire.

After this, the PCB was cleaned with acetone and the gold wire was bonded to the two copper lines. The wire should be thermally and electrically isolated from substrate and quite flat in order to cross the membrane as close as possible to its bottom surface, according to subsection 2.1. Table 1 summarizes the variations on wire distances to the substrate achieved during different fabrication processes.

Table 1. Distance of the wire crossing point in the membrane respect to the substrate in manufactured valves.

\begin{tabular}{lcccccccc}
\hline Valve sample & 1 & 2 & 3 & 4 & 5 & 6 & 7 & 8 \\
\hline Wire height $(\mu \mathrm{m})$ & 180 & 195 & 245 & 155 & 255 & 175 & 165 & 155 \\
\hline
\end{tabular}

Although wire height was controlled by a loop selector, the employed machine showed some tolerance during bonding process. Average height was approximately $190 \mu \mathrm{m}$ with a maximum deviation of $34 \%$ when compared to this value. Wire length was $1.5 \mathrm{~mm}$. The bonding process was 
carried out at $120^{\circ} \mathrm{C}$, dehydrating the PCB to improve the gold to copper connection and to enhance considerably the adhesion to the substrate. Model 4123 from K\&S Inc. was employed for this purpose.

The next step was the deposition of a thick SU-8 layer over the whole board by a spin coater SMA AC 6000 supplied from SMA Inc. The thickness of the SU-8 layer depends on the spinning speed, the time being spinning and the quantity of resist poured. To obtain high SU-8 structures with the 2150 variant the spin speed selected was $450 \mathrm{rpm}$ for 10 seconds followed by $700 \mathrm{rpm}$ during 50 seconds. With these parameters, a planar $500 \mu \mathrm{m}$ thick deposition was carried out. Although is possible to achieve membranes with higher aspect ratios the SU-8 deposition flatness is not assured.

After this, the SU-8 is baked for 15 minutes at $65^{\circ} \mathrm{C}$ in a hotplate. Then, is baked again for 180 minutes at $95^{\circ}$, avoiding exposure to visible light. Previous publications reported that lower soft bake temperatures reduces the level of stress induced into the internal SU-8 structure and prevents cracking [25]. Nevertheless, with the proposed procedure the SU-8 membranes with aspects ratios higher than 12 were fabricated without cracks or adhesion problems.

Once the resist is cured and cooled down at room temperature $\left(\sim 25^{\circ} \mathrm{C}\right)$ for 15 minutes, a high resolution transparency mask was used for lithography. SU-8 was then exposed to UV light with vacuum contact between the mask and the photoresist. A key point was the correct alignment between the gold wire and the membrane mask pattern in order to locate the resistor in the middle of the SU-8 membrane. It is remarkable that no mask aligner was needed in this step due to the tolerances provided by the device design dimensions. It is important to realize that the copper and SU-8 masks were designed for positive and negative photoresists, respectively.

When the UV exposition was finished, a post exposure bake step (PEB) was carried out, baking the board for 5 minutes at $65^{\circ} \mathrm{C}$ followed by a 10 minutes period at $95^{\circ} \mathrm{C}$ on a hotplate in order to polymerize the SU-8. After a relaxation step of 10 minutes at room temperature the following stage was to immerse the PCB with the polymerized resist in the SU-8 Developer (supplied from Microchem Corporation) for 10 minutes. This step was performed at room temperature inside a beaker using a stirring bar for slight agitation. Then the patterned SU-8 was carefully rinsed in isopropyl alcohol (IPA). Thereby, membranes with widths near to $45 \mu \mathrm{m}$ and aspect ratios of 11 were achieved with nearly perfect vertical profiles.

After PEB and cleaning, a $0.8 \mathrm{~mm}$ diameter orifice was drilled in the chamber through the PCB to connect an external pump and set up the working pressure. Drilling dust was removed and the SU-8 cavities cleaned with IPA.

A thin SU-8 2025 layer was spinned over the methaclylate cover, which was previously cleaned with IPA. The spin speed was selected to $500 \mathrm{rpm}$ for 5 seconds followed by $3000 \mathrm{rpm}$ for 40 seconds, resulting in a $30 \mu \mathrm{m}$ thick deposition. The thickness of this layer was selected in order to avoid undesirable displacements into the cavities of the resist in contact with the device walls, which could cover the gold wire making its thermal actuation worse or obstruct the fluid flow though the valve. A detailed study of this phenomenon is widely reported in [26].

The cover bonding process with the microvalve structure was carried out in absence of pressure applied over the methaclylate board, when the cover weight causes the uniform distribution of the spinned SU-8 on the microvalve SU-8 walls. Thus, the resist located on the cover diffuses over the structure, ensuring the device enclosure and preventing possible pressure leakages in the chamber. The bonding is carried out by means of an UV exposure step of 1.5 minutes over the whole device, achieving a very strong cross-linking between both SU-8 resists. The fabricated device is shown in figure 8. An overhead photograph of the membrane is illustrated in figure 9 . 


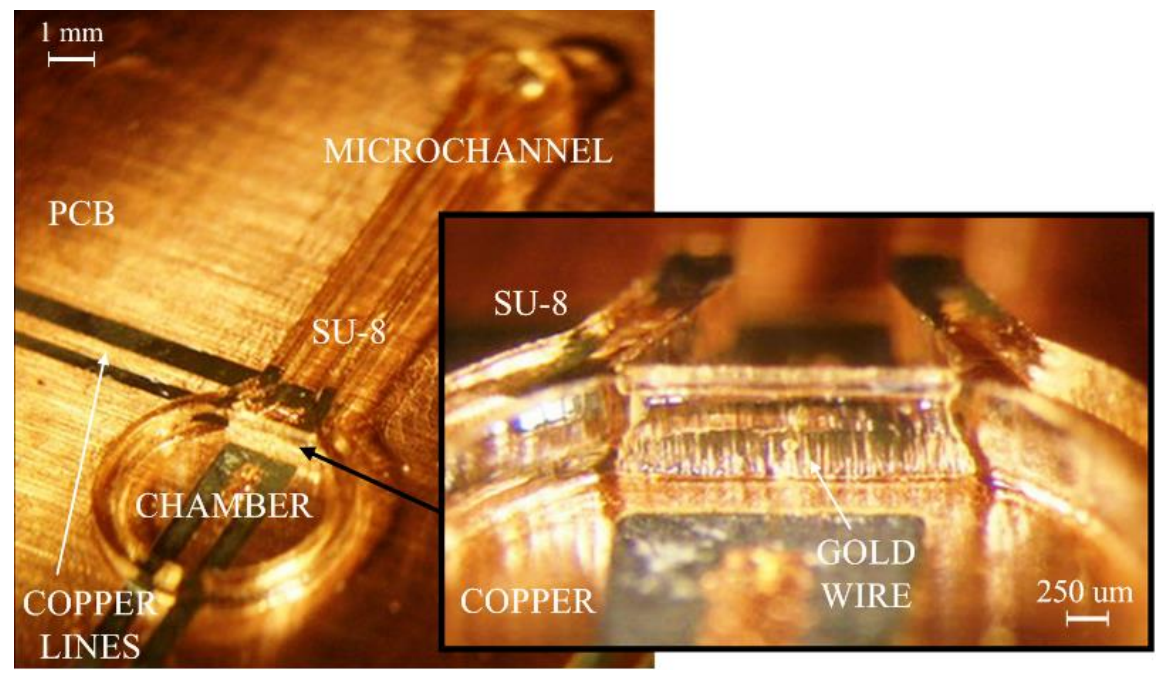

Figure 8. Photograph of the microvalve and enlarged view of the SU-8 membrane with the gold wire through it.

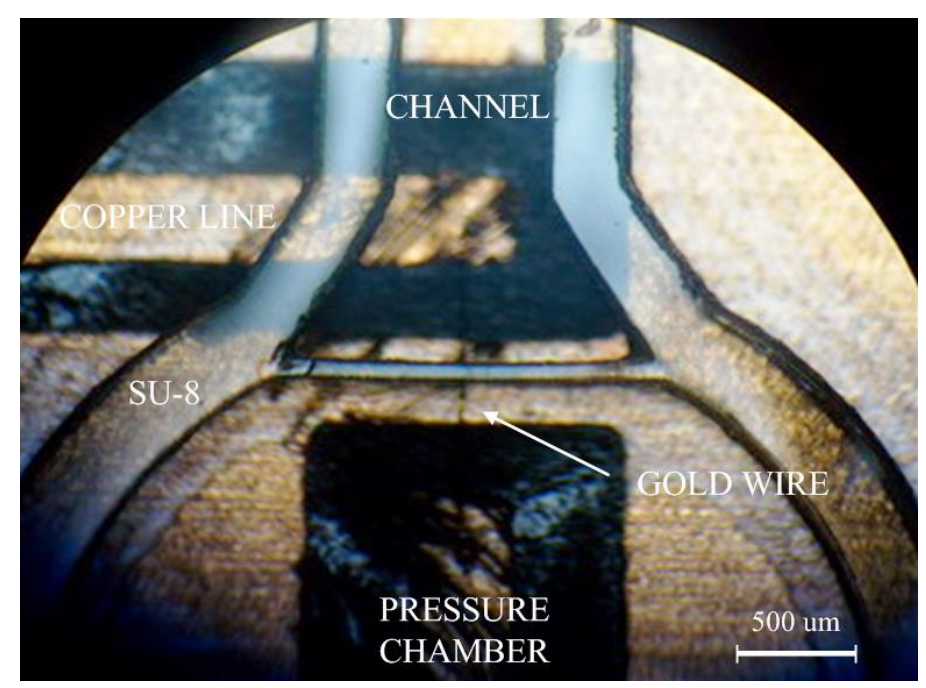

Figure 9. Overhead photograph of the membrane.

\section{Experimental tests}

Once the microvalve was fabricated experimental test were carried out to confirm the correct operation of the device. The goal is to study the membrane behaviour at different pressures when a current is applied through the gold wire. With these results, the proposed microvalve will be characterized, confirming the relation between theoretical studies and experimental outcomes.

\subsection{Experiment setup}

Following the fabrication process described in section 3 , several microvalves with membrane aspects ratios of 4:1, 9:1 and 11:1 (height:width) were produced and prepared for pressure and temperature assays. An optimal balance between power consumption and pressure required will be necessary to optimize the microvalve operation.

The device chamber was connected to an external air pump to fix a suitable pressure in it according to a pressure gauge. A control valve regulated the maximum pressure selectable up to $0.8 \mathrm{MPa}$. The microchannel fluid port was set at atmospheric pressure and coupled to a small tank containing black 
ink diluted in water, in order to inspect the fluid flow along the device when the activation was performed. Two external wires connected the copper lines to a DC source to supply the current pulse through the gold wire during experiments. The whole assembly is illustrated in figure 10. A microscope connected to a camera provided a real-time inspection of the device behaviour through a personal computer screen.

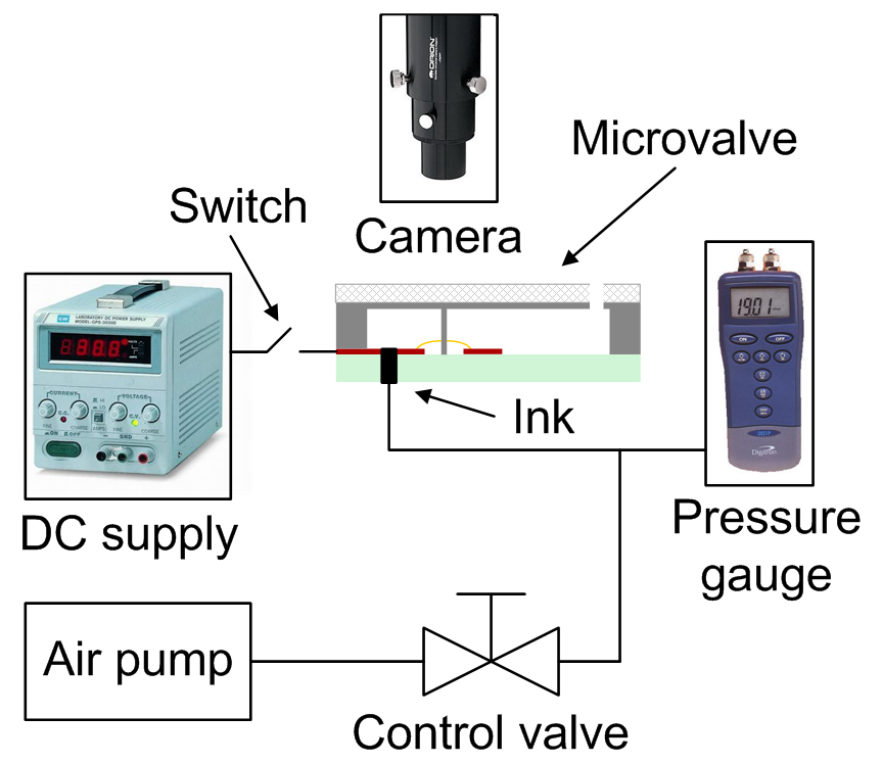

Figure 10. Experimental setup for microvalve testing and characterization.

\subsection{Results}

Pressure was progressively supplied to the microvalve chamber in absence of current until reaching the desired value. Working pressure was maintained for 1 minute approximately in order to avoid undesired transient effects. It was verified that the maximum pressure the device could withstand without leakages was above $0.8 \mathrm{MPa}$. At that moment, electric current was gradually incremented through the gold wire until valve activation was achieved. The detailed process steps are illustrated in figure 11 .

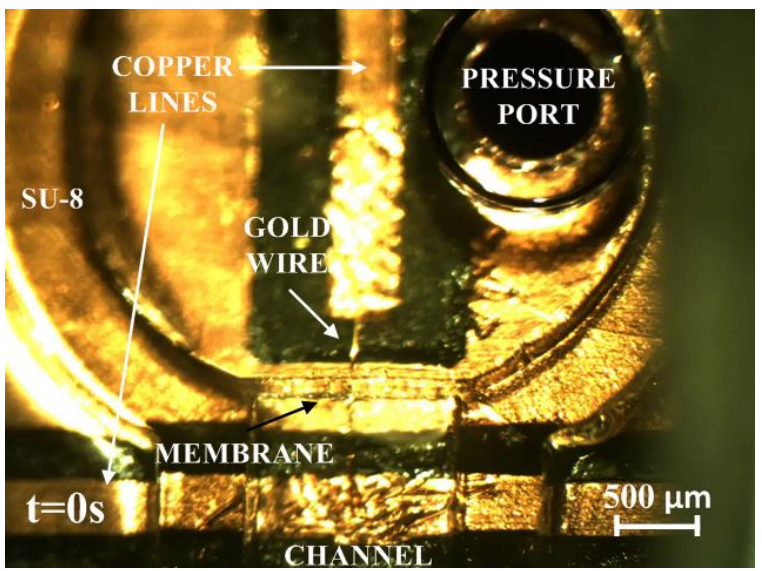

(a)

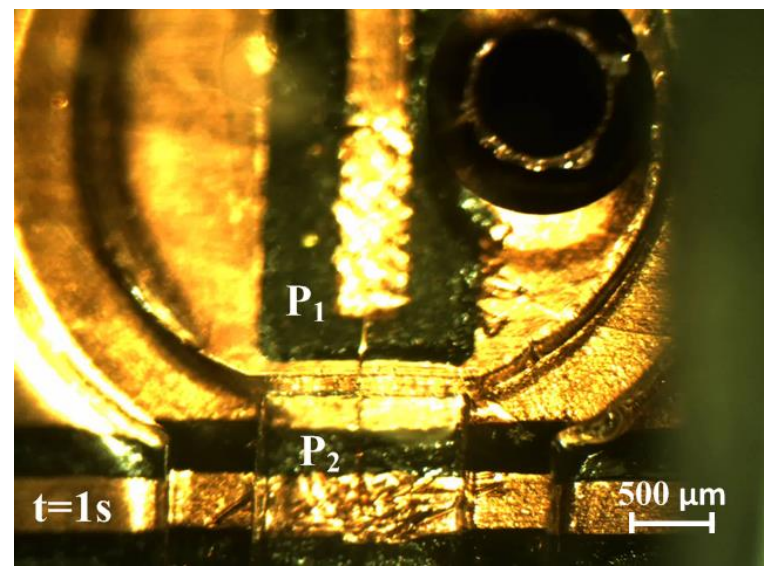

(b) 


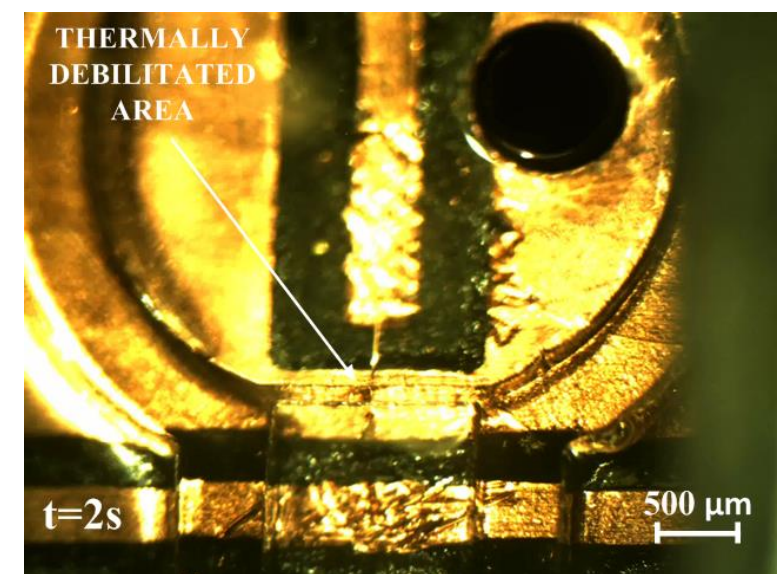

(c)

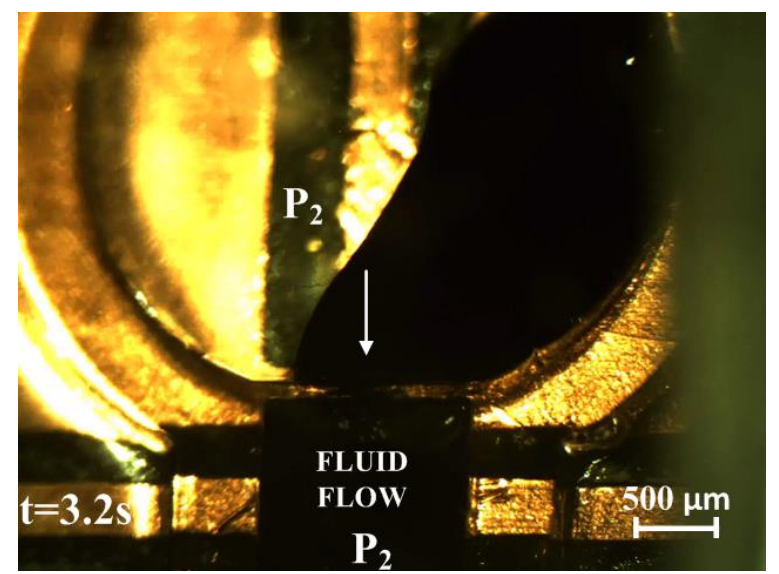

(d)

Figure 11. Microvalve operation process. (a) Valve photograph with components description ( $\mathrm{t}=0 \mathrm{~s}$ ). (b) Pressure in chamber is fixed to $P_{l}$. The membrane supports the pressure applied and the current is gradually incremented $(\mathrm{t}=1 \mathrm{~s})$. (c) DC current is still connected through the gold wire. The membrane starts to weaken due to the thermal power contribution $(t=2 s)$. (d) The membrane finally breaks and the fluid is pushed from the chamber to the microchannel $(\mathrm{t}=3.2 \mathrm{~s})$.

Photograph 11(b) shows the membrane supporting a pressure difference of $0.5 \mathrm{MPa}$. When pressure is fixed and current is supplied, wire temperature increases and starts debilitating the membrane surrounding area, as is shown in photograph 11 (c). A slight membrane displacement is observed in this area due to the bending stress induced by the pressure difference between chamber $\left(P_{l}\right)$ and microchannel $\left(P_{2}\right)$. After a few seconds, pressure finally breaks through the lower centred point of the membrane resulting in a fluid flow towards the microchannel, as is illustrated in photograph 11 (d). For the reported experiment a valve with 11:1 membrane aspect ratio was selected, achieving activation in 3.2 seconds. Table 2 reports the activation energy for each inlet pressure applied, which was calculated as the time integral of the electric power supplied to the wire.

Table 2. Different activation conditions for the 11:1 aspect ratio microvalve.

\begin{tabular}{lccccc}
\hline $\begin{array}{l}\text { Inlet pressure } \\
(\mathrm{MPa})\end{array}$ & $\begin{array}{c}\text { Average } \\
\text { current } \\
(\mathrm{A})\end{array}$ & $\begin{array}{c}\text { Voltage } \\
(\mathrm{V})\end{array}$ & $\begin{array}{c}\text { Applied } \\
\text { power } \\
(\mathrm{W})\end{array}$ & $\begin{array}{c}\text { Time } \\
\text { response } \\
(\mathrm{s})\end{array}$ & $\begin{array}{c}\text { Energy } \\
(\mathrm{J})\end{array}$ \\
\hline 0.1 & 1.65 & 0.65 & 1.07 & 3.0 & 3.21 \\
0.3 & 1.40 & 0.55 & 0.77 & 2.5 & 1.92 \\
0.5 & 0.90 & 0.38 & 0.34 & 3.2 & 1.09 \\
0.7 & 0.80 & 0.35 & 0.28 & 2.5 & 0.70 \\
\hline
\end{tabular}

This experiment was also carried out with the remaining groups of valves divided in 9:1 and 4:1 aspect ratios. Figure 12 illustrates pressure and heat required in the wire to activate these aspect ratio devices. 


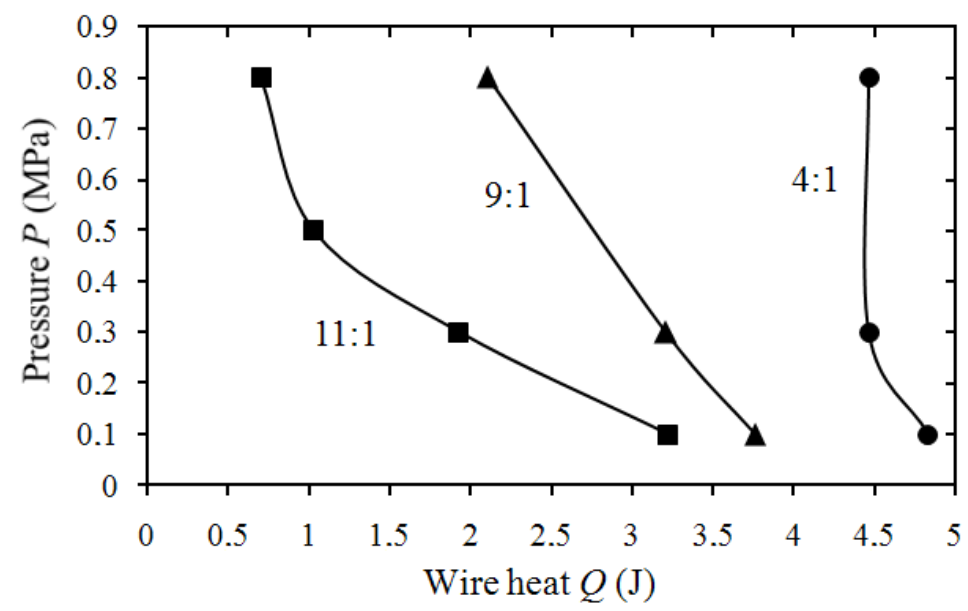

Figure 12. Heat dissipated by the wire to activate the valve as a function of the operation pressure for different membrane aspect ratio designs.

As shown in the graphic, very low membrane aspect ratios force a high heat contribution due to the slight bending stress caused by applied pressure. Curve 4:1 illustrates that great widths in the membrane dimensions will minimize the pressure effect over the structure. For medium aspect ratios (9:1) the thermal energy required for activation increases linearly with pressure, suggesting a similar thermal and mechanical contribution. Different slopes are visualized for curve 11:1 where higher pressures cause slight variations in the heat required, indicating a more important mechanical contribution in activation process than thermal. Figure 12 also illustrates that for a given pressure in the valve chamber, higher temperatures are required as lower membrane aspect ratios are selected. Microvalve operation zones will be discussed in detail in the next section.

\section{Discussion}

The different operation zones that theoretical study has suggested in device behaviour according to the experimental results shown for the best performance microvalve are now discussed. Figure 13 illustrates these microvalve operation zones for a membrane aspect ratio of 11:1.

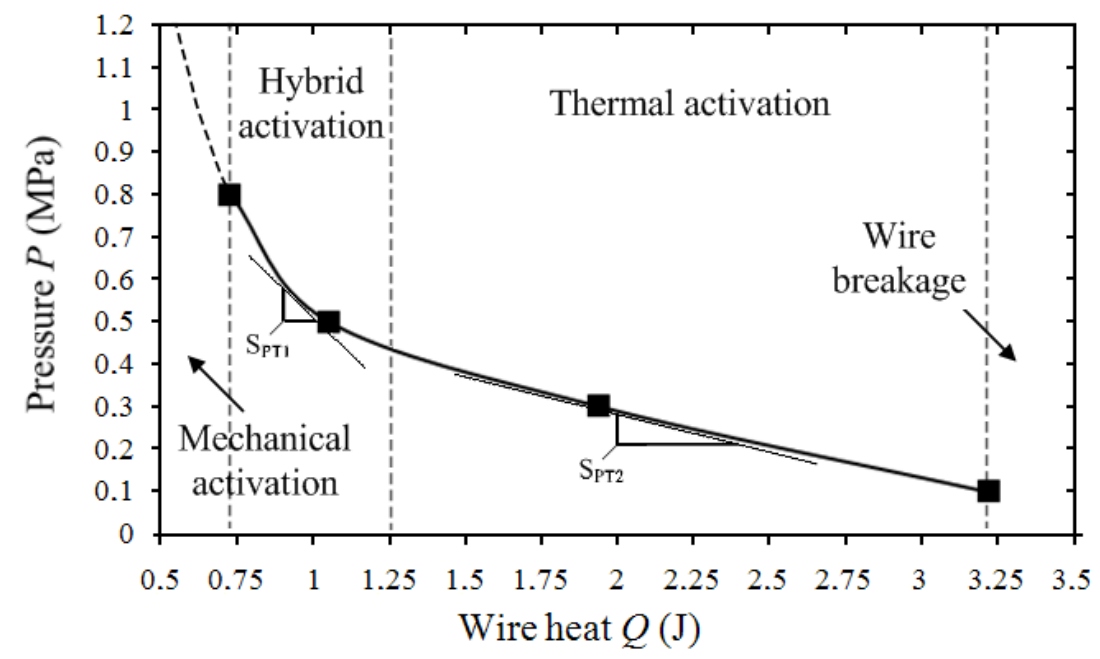

Figure 13. Device operation zones as a function of the wire heat and the pressure supplied to the membrane. 
The operation zones as a function of the pressure applied are listed below:

- Mechanical activation: When the current supplied is very low or zero the mechanical activation principle will be the only responsible for membrane breakage. The heat generated in the wire will be not enough to cause a significant thermal weakening for the SU-8 membrane. Thus, activation moment will be impossible to control without a thermal trigger.

- Hybrid activation: In this operation range the breakdown of the membrane will be achieved by a hybrid actuation, thermal and mechanic. The wire heat reduces the SU-8 fracture strength and the membrane model as a rectangular plate under a uniform pressure is still valid. The mechanical stress will be the principal cause of activation and the thermal contribution will be consigned to a secondary level. Hence the actuation will be carried out mechanically, acting the temperature increasing as a trigger for activation.

- Thermal activation: When heat increases and wire temperature goes beyond $200{ }^{\circ} \mathrm{C}$ the SU-8 reaches its glass transition point and the theoretical model of a viscous cylinder flow must be considered. The thermal contribution will be dominant then over mechanical which leads to a high power consumption of the device. According to the slow curve slope observed, high pressures will just decrease linearly the valve time response. When temperature is around 380 ${ }^{\circ} \mathrm{C}$ the SU-8 from the membrane starts to degrade and physical properties drastically change.

- Wire breakage: When the heat generated induces a wire temperature increase close to the gold melting point, the wire melts down disabling the device functionality.

From the point of view of minimizing the electric power consumption, the pressure contribution must be dominant over the thermal contribution in order to break the membrane and cause the fluid flow. With that in mind, the hybrid activation zone corresponding to a pressure-dependent behavior was the optimal operation area, with an energy required for activation typically of $0.7 \mathrm{~J}$.

According to the graphic shown in Figure 13, the relation between the supplied heat $Q$ and the inner chamber pressure $P$ required for valve activation can be defined as follows:

$$
S_{Q P}=\frac{\partial Q}{\partial P}
$$

This parameter expresses the variation of the heat required in the resistor when the chamber pressure changes. $S_{Q P}$ is negative all over the working range due to an increase of pressure will always reduce the thermal energy required to activate the valve. In hybrid activation zone, the device requires less thermal contribution when pressure varies, considerably reducing the electrical power consumption. The most important microvalve nominal characteristics that have been measured are summarized in Table 3.

Table 3. Microvalve nominal characteristics.

\begin{tabular}{ccccc}
\hline $\begin{array}{c}\text { Membrane } \\
\text { aspect ratio } \\
\text { (height:width) }\end{array}$ & $\begin{array}{c}\text { Maximum working } \\
\text { pressure } P \\
(\mathrm{MPa})\end{array}$ & $\begin{array}{c}\mathrm{S}_{\mathrm{QPmax}} \\
(\mathrm{J} / \mathrm{MPa})\end{array}$ & $\begin{array}{c}\text { Maximum time } \\
\text { response } t \\
(\mathrm{~s})\end{array}$ & $\begin{array}{c}\text { Minimum energy } \\
\text { required } Q \\
(\mathrm{~J})\end{array}$ \\
\hline $11: 1$ & 0.8 & -1.6 & 3.2 & 0.7 \\
\hline
\end{tabular}

Similar microvalves have been previously reported by different authors, presenting a combined activation process by thermal and mechanical principles. Guerin's group developed a single-use microvalve that included a heating resistor which melted and delaminated a PE/PET membrane from the substrate [27]. In a similar way to the work described in this paper, an overpressure existing behind the membrane forced the valve to open, requiring a power about 200 to $400 \mathrm{~mW}$ for $1.2 \mathrm{~s}$. Although 
this design presented a lower energy required for operation, it was only capable of withstanding a pressure of around 0.2 $\mathrm{MPa}$, resulting insufficient for high pressure-driven applications.

Another device similar to the described here is the work reported by Debray et al [17], where the valve consisted in a channel obstructed by a membrane coated with a low melting point alloy. At $20^{\circ} \mathrm{C}$ the alloy was solid and could withstand an input pressure higher than $3 \mathrm{MPa}$, but the device opened 33 $\mathrm{s}$ after the temperature had reached $47{ }^{\circ} \mathrm{C}$, presenting a relatively slow time response. For the irreversible paraffin-based microvalves [16] time responses are also slow (on the order of tens of seconds) with a maximum holdup pressure around $275 \mathrm{kPa}$. Nevertheless, the power required for actuation is close to the design presented in this paper $(100-200 \mathrm{~mW})$.

Mueller et al [28] have developed a single-use valve for micro-propulsion applications whose actuation principle is based on a silicon membrane that withstands pressures up to $20 \mathrm{MPa}$. The main drawback is the high temperature required to melt the membrane $\left(1400{ }^{\mathrm{a}} \mathrm{C}\right)$, which is not compatible with many applications. Finally, Bejhed et al [18] presented a robust microvalve for high pressure space applications, capable of being functional at pressures of $10 \mathrm{MPa}$. An important innovation of this design was the incorporation of a filter which served to remove any particle debris created by the activation process. In these studies, the valve opened in less than $10 \mathrm{~s}$ but required an applied power of $13 \mathrm{~W}$.

Regarding the use of the microvalve in biomedical applications, the contact between the fluid and the membrane should be avoided, due to the high temperatures reached around the gold wire. Most of the biological fluids are not compatible with temperatures near $200{ }^{\circ} \mathrm{C}$, resulting in a degradation of the analyte properties of interest. Besides this, the contact between the viscous SU-8 from the membrane and the biological fluid may also affect its properties and consequently the measurements performed. Nevertheless, some little debris from the membrane may be released to the fluid so a safety area must be kept in order to avoid an undesirable mixing. Although this matter is currently being studied, some strategies are described as follows.

When chamber pressure is higher than atmospheric, the valve will operate as a microinjector, pushing the fluid through the microchannel to the output port (Figure 6). In this case, the fluid would be contained in a reservoir located in the microchannel path separated from the membrane and the gold wire. Thus, the chamber pressure will drive the fluid from the microchannel reservoir to the output port when the SU-8 membrane is broken, preventing the contact between the membrane and the fluid. A safety distance between the fluid reservoir and the membrane must be determined in order to avoid the fluid warming when an electric current is supplied to the gold wire. However, this safety distance would be short because the high temperature area is located close to the membrane due to the small dimensions of the wire.

When the valve operates in microextractor mode, chamber pressure will be higher than atmospheric and fluid will flow through the microchannel towards the broken membrane. Dimensions of the microchannel must be calculated to ensure the channel filling with fluid except the safety area around the membrane. A suitable place to include a biochemical sensor to analyze biological substances in the fluid is in the microchannel, close to the input where fluid filling is ensured when valve is activated. This way, membrane temperature or debris is far enough to affect the sensor measurements.

\section{Conclusions}

A single-use microvalve for pressure-driven microfluidic applications has been presented. By means of a hybrid thermal and mechanical actuation principle, low power consumption has been achieved. The main advantages of this device lie on its simplicity, robustness, great functionality and ease of integration due to its planar structure, improving previously reported single-use valves due to its simple fabrication process and low cost based on SU-8 and PCBMEMS technologies.

Experimental results have proven the microvalve functionality, working in a pressure range up to $0.8 \mathrm{MPa}$ and presenting an energy consumption of $0.7 \mathrm{~J}$ for less than 3.2 seconds to be open.

The device has been conceived as a single component of a microvalve array platform, being a key part of an integrated microfluidic Lab-on-chip. Valve portability can be improved by adapting a small 
battery with an activation switch and adding a pressurization extra step in fabrication process. Future works also include integration of microneedles and biochemical sensors to analyze biological substances.

\section{Acknowledgment}

The described investigations were carried out within the research project MEDITRONIC granted by the Junta de Andalucía (Spain) (P06-TEC-01561). Francisco Perdigones and Carmen Aracil from University of Seville Electronic Engineering Department are also acknowledged for helpful comments in theoretical simulations and experimental assays.

\section{References}

[1] Tabeling P 2005 Introduction to Microfluidics (Oxford University Press)

[2] Vilkner T, Janasek D and Manz A 2004 Micro total analysis systems. Recent developments Anal. Chem. 76 3373-86

[3] Geschke O, Klank H and Telleman P 2004 Microsystem Engineering of Lab-on-a-Chip Devices (Wiley Press)

[4] Thorsen T, Maerk1 S and Quake S R 2002 Microfluidic large-scale integration Science 18 580-4

[5] Kim D S, Lee S H, Ahn C H, Leed J Y and Kwon T H 2006 Disposable integrated microfluidic biochip for blood typing by plastic microinjection moulding Lab on Chip 6 794-802

[6] Boden R, Lehto M, Margell J, Hjort K and Schweitz J 2008 Onchip liquid storage and dispensing for lab-on-a-chip applications J. Micromech. Microeng. 18 IOP 075036

[7] Saliterman S S 2006 Fundamentals of BioMEMS and Medical Microdevices (SPIE Press)

[8] Kwang W and Ahn C H 2006 A review of microvalves J. Micromech. Microeng. 16 R13-R39

[9] Zhang C, Xing D and Li Y 2007 Micropumps, microvalves, and micromixers within PCR microfluidic chips: Advances and trends Biotechnology Advances 25 483-514

[10] Bohl B, Steger R, Zengerle R and Koltay P 2005 Multi-layer SU-8 lift-off technology for microfluidic devices J. Micromech. Microeng. 15 1125-30

[11] Becker H and Gartner C 2000 Polymer microfabrication methods for microfluidic analytical applications Electrophoresis 21 12-26

[12] Shaw J M, Gelorme J D, LaBianca N C, Conley W E and Holmes S J 1997 Negative photoresists for optical lithography IBM J. Research Develop. 41 81-94

[13] Mata A, Fleischman A J and Roy S 2006 Fabrication of multi-layer SU8 microstructures $J$. Micromech. Microeng. 16 276-284

[14] Voskerician G,Shive M S, Shawgo R S, Recum H, Anderson J M, Cimac M J and Langer R 2003 Biocompatibility and biofouling of MEMS drug delivery devices Biomaterials 24 1959-67

[15] Wego A, Richter S and Pagel L 2001 Fluidic microsystems based on printed circuit board technology J. Micromech. Microeng. 11 528-31

[16] Liu R H, Yang J, Lenigk R, Bonanno J and Grodzinski P 2004 Single-use, thermally actuated paraffin valves for microfluidic applications Sensors Actuators B 98 328-36

[17] Debray A, Shibata M and Fujita H 2007 A low melting point alloy as a functional material for a one-shot micro-valve $J$. Micromech. Microeng. 17 1442-50

[18] Bejhed J, Rangsten P and Kohler J 2007 Demonstration of a single use microsystem valve for high gas pressure applications J. Micromech. Microeng. 17 472-81

[19] Ahn C H, Choi J W, Beaucage G, Nevin J H, Lee J B, Puntambekar A, Lee J Y 2004 Disposable smart lab on a chip for point-of-care clinical diagnostics Proc. IEEE 92 154-73

[20] Young W C and Budynas R G 1989 Roark's Formulas for Stress and Strain (McGraw-Hill)

[21] Schoeberle B, Wendlandt M and Hierold C 2008 Erratum to Long-term creep behavior of SU-8 membranes: Application of the timestress superposition principle to determine the master creep compliance curve Sensors Actuators A 148 352-3

[22] Feng R and Farris R J 2003 Influence of processing conditions on the thermal and mechanical 
properties of SU8 negative photoresist coatings J. Micromech. Microeng. 13 80-88

[23] Namazu T, Inoue S, Takio K, Fujita T, Maewzka R and Koterazawa K 2005 Visco-elastic properties of micron-thick SU-8 polymers measured by two different types of uniaxial tensile tests Micro Electro Mechanical Systems 2005 18th MEMS IEEE International Conference pp 447- 50

[24] Moreno M, Aracil C, Quero J M 2008 High-integrated microvalve for lab-on-chip biomedical applications IEEE Biomedical Circuits and Systems Conference pp 313-16

[25] Anhoj T A, Jorgensen A M, Zauner D A and Hübner J 2006 The effect of soft bake temperature on the polymerization of SU-8 photoresist J.Micromech. Microeng. 16 1819-24

[26] Merkel T, Graeber M and Pagel L 1999 A new technology for fluidic microsystems based on PCB technology Sensors Actuators A 77 98-105

[27] Guerin L J, Dubochet O, Zeberli J F, Clot P and Renaud P 1998 Miniature one-shot valve Micro Electro Mechanical Systems 1998 11th Anual International Workshop pp 425- 28

[28] Mueller J, Marrese C, Polk J, Yang E H, Green A, White V, Bame D, Chadraborty I and Vargo S 2003 An overview of MEMS-based micropropulsion developments JPL Acta Astronaut. 52 881-95 